\title{
Smart Parking for Smart Cities using loT
}

\author{
Bhavani Ratakonda ${ }^{1}$, Pavithra Avvari ${ }^{2}, B$. Rajarao $^{3}$, Voruganti Sreevani ${ }^{4}, N$ V Ganapathi Raju ${ }^{5}$
}

\begin{abstract}
${ }^{1}$ Assistant Professor, Department of IT,Gokaraju Rangaraju Institute of Engineering and Technology, Hyderabad, India ${ }^{2}$ Assistant Professor, Department of IT,Gokaraju Rangaraju Institute of Engineering and Technology, Hyderabad, India

${ }^{3}$ Assistant Professor, Department of CSE, Mallareddy Engineering College, Hyderabad, India.

${ }^{4}$ Assistant Professor, Department of CSE, Mallareddy Engineering College, Hyderabad, India

${ }^{5}$ Professor, Department of IT, Gokaraju Rangaraju Institute of Engineering and Technology, Hyderabad, India
\end{abstract}

\begin{abstract}
The idea of smart cities has gained immense popularity in real-time, Internet of Things (IoT) is not just about connecting people and about connecting things. Billions of devices will connect to the internet through sensors they talk to each other. Searching for parking is a routine activity for many peoples in the city around the world. There are many solutions some of the existing solutions are inductive loops, image processing at shopping malls, universities, airports,also, the thought behinds advancement of IoT. With this IoT there is numerous wide scope of utilizations in our day by day life, not many of them are keen urban communities, smart waste administration, smart health, brilliant security, and smart industry applications. This paper provides a novel solution for the people to minimize the waiting time and maximize productivity and help to find the space where the vehicles can be left to decrease the traffic in the leaving region.Internet of Things and distributed computing are two unique parts of innovations. IoT is an arrangement of interrelated gadgets and things with unique identifiers associated with the web can speak with one another. The cloud gives everything as administration i.e. pays for what we use. The obstacle sensor senses the obstacle status when the car is being parked in the parking area and status is sent to the cloud. Utilizing the IoT framework it gives the accessible free stopping spaces, Entry and exit likewise gives payment process through RFID card to diminish the bottleneck of the payment procedure and approve at the passage/leave the entrance of the shopping area.
\end{abstract}

\section{Introduction}

The Internet of Things is a stable network infrastructure of enclosed objects/gadgets with identifiers in which $\mathrm{m} 2 \mathrm{~m}$ communication with less human intervention is recognized, which is conceivable using generic and interoperable conventions[1]. The 'thing' in IoT could be objects that have been provided an IP address and have the capability without manual intervention to capture and transmit data over a network.[14]

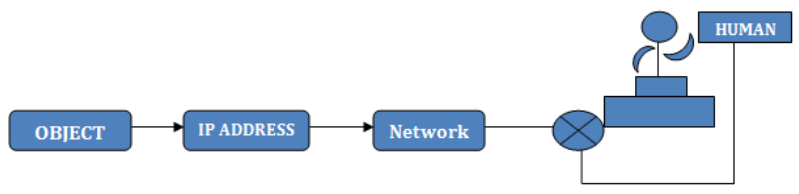

Fig.1. Thing behaves in IoT

More than half of the world population lives in urban areas so the cities have reached their full occupancy. Gadgets developed in various frameworks can be connected to the web through IoT.[14] They can be managed from anywhere in the instant when devices can talk to themselves digitally. At that point, the challenges are emerging us to collect more data from more sites, enabling more ways to increase efficiency and enhance well-being and IoT protection [2].

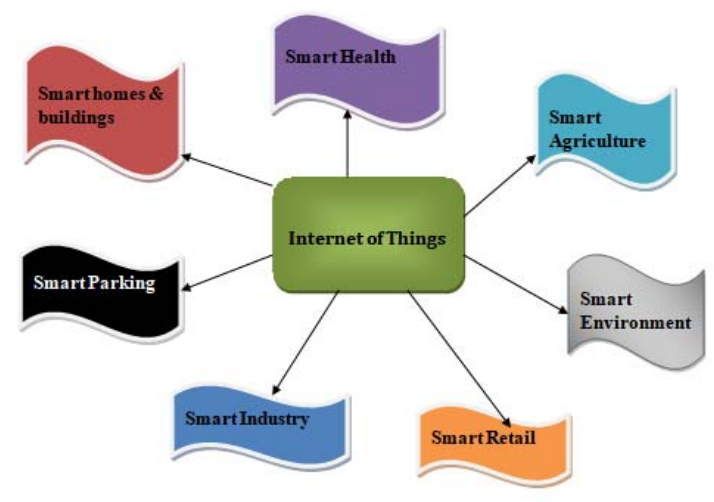

Fig.2. Applications of IoT

There are very wide varieties of applications that can come across in our daily life: 
1) Smart homes and buildings:

With the IoT application developed, the user can control the functionality of home appliances with just one touch like adjustment of $\mathrm{AC}$ temperatures in your bedroom before you even reach home, Set an alarm so that you are never late for the important things in life, Enjoying movie nights by setting your favorite movie lists on TV and never miss out on your grocery list.[14] IoT can assist in the design of well-groomed management of home power use, computer communication, emergency detection, house security measures, and easy finding of items, home security, etc.[15]

\section{2) Smart Health:}

Smart wellbeing is one of the uses of IoT. With this technology in the health sector, those devices like the health band can be capable to save life or improve the quality of life.Ex: Checking healthiness parameters, support for free-living, observing medicine consumption, and so forth.[14]

\section{3) Smart Agriculture:}

Various sensors of a network can able to detect information so that it can easily carry out data processing and notify easily to the farmer from endtoend communication infrastructure. For example, the portion of land that needs special attention could be sent with the assistance of cell phone text messages. This may incorporate well-groomed wrapping of seeds, compost, and vermin control components that react to explicit neighborhood conditions.[15] A smart cultivating framework will assist agronomists with having upgraded keen of the plant development models and effective cultivation by obtaining data on soil conditions and changeability in the atmosphere. This would create rural proficiency by staying away from the unseemly cultivating conditions.

\section{4) Smart Environment:}

It includes waste management, air pollution, and water monitoring, etc. Water Scarcity observing is the primary concern that must be concentrated. This IoT will make it easier to spot the shortage of resources at various locations.In addition to monitoring, long term water interventions such as groundwater flow management, sensor networks connected to the specific simulation activities may also be used to notify people of a stream, for example, if an upstream occurrence, the accidental arrival of sewage into the stream, can still have risky implications.[15]

\section{5) Smart Retail:}

Forecast of natural disasters is one of the issues. The game plan of sensors and their self-guided association and reenactment will assist with figuring likelihood of landslides or other catastrophic events and to take proper action in advance.

\section{6) Smart Manufacturing:}

In the technology, IoT will discover applications., dealing with an armada of vehicles for an organization. IoT assists with observing their natural execution and procedure with the information to decide and pick one that needs upkeep.

\section{7) Smart Parking:}

Smart parking is a part of transportation. This uses detecting gadgets, for example, cameras, vehicle tallying equipment, sensors introduced in pavements, and so on to determine occupancy of the parking slot. The framework builds the accessibility of parking with the utilization of sensors. It keeps the drivers from investing an excess time for searching a parking speck. We are moving towards a general public with the advancement of innovation; where each and everyone will be interconnected. Because of this, a large portion of the individuals invests their significant time in looking through leaving openings to leave their vehicles. Here answering the parking system by utilizing cloud put together in a framework concerning expertise innovations. The idea of this began with things of identification of communication gadgets. The gadgets could be followed, controlled by utilizing mobile application are associated through the web. IoT enhances the use of the internet to provide communications [3]. Internet" and "things" are the two conspicuous terms in IoT.[15] The Web implies an enormous worldwide infrastructure of associated servers, PCs, tablets, and mobiles that use conventions and design frameworks globally. The Web allows data to be submitted and acknowledged. As a rule, IoT involves the interconnection of gadgets and physical artifacts, several objects can collect information in remote areas and provide information in procedures and services to units that track, protect, sort, and analyze the information. The module includes the obstacle sensors which sense the blockage condition when the car is parked in the parking area and it sends the status to the cloud. Using the IoT system provides the available slots. It includes the RFID card-based entry/exit. The payment is also processed through an RFID card to reduce the difficult situation at the entry or exit gates of the parking slot [4].

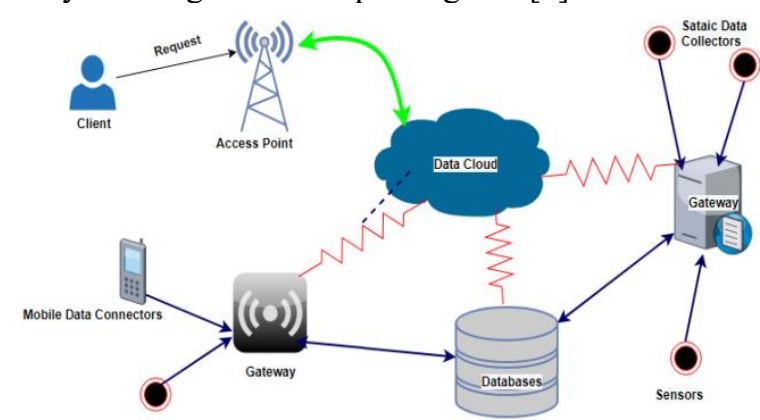

Fig.3. Parking System Interaction with Cloud

\section{Literature survey}

The present world is of the internet which is making our lives simpler just as quickly. The utilization of advanced cells has incredible applications. The IoT stages permit us to interface the sensors through the internet. The parking structure is one of them. There are numerous 
issues in India as well as in the rest of the world due to spontaneous parking. Thus we are attempting to present the parking framework dependent on IoT to make the voyaging at ease. The portable applications are very easy to use interfaces can give better outcomes for parking slots places even though this data isn't given to the clients about free openings accessibility. This results in heavy traffic, time consumption, and causes contamination. The data in regards to accessible parking spots in the purpose of the spot can be resolved with this procedure called Smart Parking System.It includes with ease sensors, continuous information assortment, and cell phone-empowered customized payment frameworks that license individuals to keep down parking ahead of time to precisely anticipate the specific spot where there is probability. Smart Parking encourages perhaps the most serious issue on driving in urban zones, discovering void parking spots, and controlling prohibited parking. Generally, this innovation is tied in with improving the productivity and administration levels of responsibilities in gathering and interpreting the information at the Street level.[5]

\subsection{Existing System}

Ongoing increment in the development of the car industry combined with the endless attention of PCs asked the requirement for better and more intelligent parking methodology. The issue in the current framework is in the case of parking space is accessible or not driver doesn't know before arriving at the parking place. Labor is required and we can't find the specific area of the empty parking opening. In many current systems, the driver must drive around the parking area to locate the space. The vast majority of the current leaving the executives frameworks seldom to fix the issues of spot board parking, the direction of the car, reservation of parking garage, and so on.

\subsection{Proposed System}

This paper proposed a Smart Parking reservation framework utilizing short message services that give sophisticated highlights like remote parking inspection, computerized direction, and parking declaration system. The paper depicts the general framework design of SPARK from equipment to programming execution in the view purpose of sensor systems. We actualized a complete model for parking organizations to understand the structure functionalities and highlights referenced. Here we use IR sensors for checking and screening the status of the vehicle leaving in LCD. On the off chance that someone wants to reserve parking space by sending SMS to a specific area, the GSM modem receives the message in the parking zone and takes care of the microcontroller by using UART correspondence. The microcontroller takes the requested message and, with the aid of an IR sensor, checks the accessibility of a location in the parking area. [6]. If it is no vacant lot available, Other controllers respond to the unavailable status of the requested mobile number using GSM technology concerning the time of arrival at the parking area. With the help of this methodology, one can easily locate all possible areas of parking to avoid traffic congestion and it minimizes cost and time. Anybody can notice the parking spot from anyplace by utilizing the versatile application. In this framework, the propelled sensors are utilized to locate the free opening and transferred the information to the IoT. The user will receive information on a parking location, the number of parking openings, and all other data on feasible parking areas.

\section{WORKING PRINCIPLE}

In the traditional process, the devices are expensive and do not connect to a user's. In this proposed system we are using ARDUINO microcontroller which is the prime controller. Here we are utilizing different modules, for example, Ultrasonic sensor, RFID Reader and Tag, LCD. The ultrasonic sensor faculties for interruption present in the parking opening. In the same slot when the car is located, the anticipation sensor detects the vehicle is available are not and once the vehicle recognized the space, full status is sent to the cloud. When the RFID card is shown the card details will be displayed on the LCD screen and the amount will deduct for the car from the total [7][12].

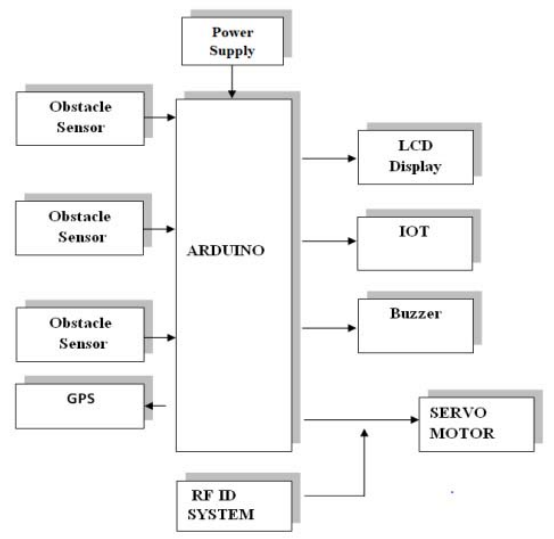

Fig.5. Block Diagram

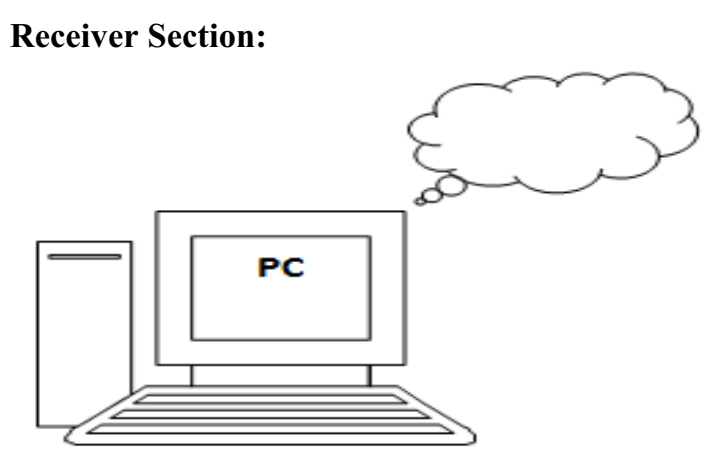

Fig.6.Receiver Section On Pc

\section{WORKING:}

Stage 1:

The principle preferred position of the present framework is the client will enroll application/site. In this application, it will show the data of parking spaces exclusively. 


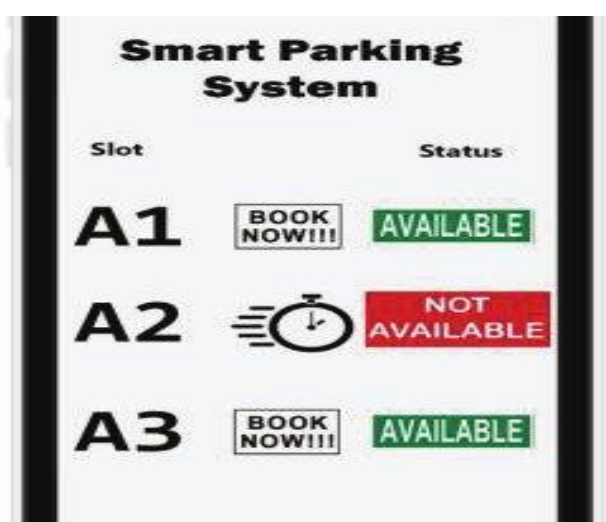

Fig.7. Parking Slot Availability

\section{Stage 2:}

The user uses the RFID card for validation then the servo motor opens the gate automatically after the successful validation. When the car enters the parking area IR sensor occurrence will detect the parked vehicle and updates the data to the cloud automatically.

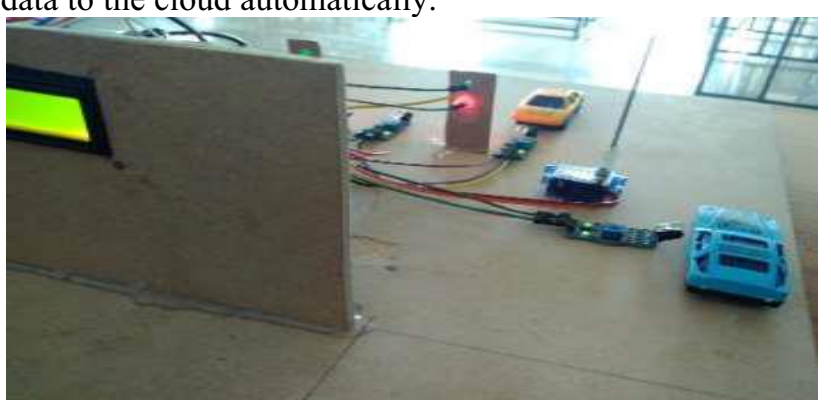

Fig.8. Parking the vehicle in a shopping area

\section{Stage 3:}

The vehicle will go into the parking place around then the individual doesn't realize which slot is vacant, for this there will be a sign of LEDs for each space when the greenlight sparkles the space is vacant when the red light turns on the space was filled. With this, every individual effectively realizes which space is vacant.

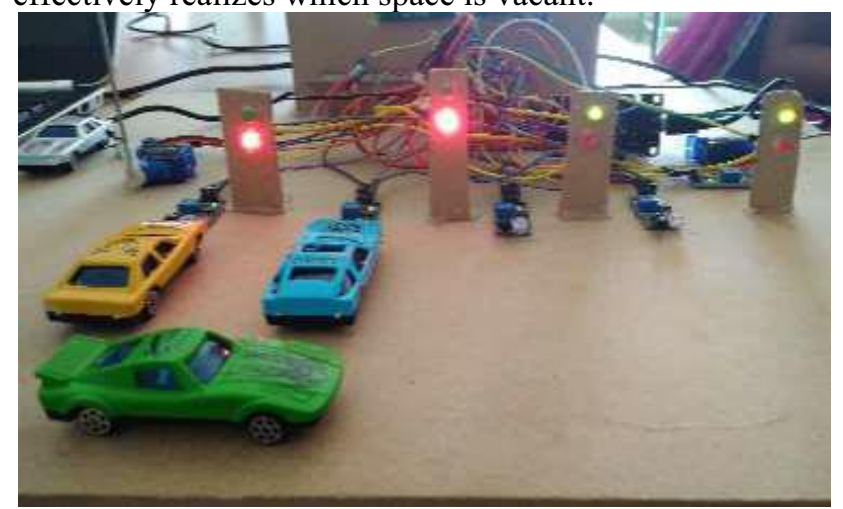

Fig.9.Before reaching to the slot

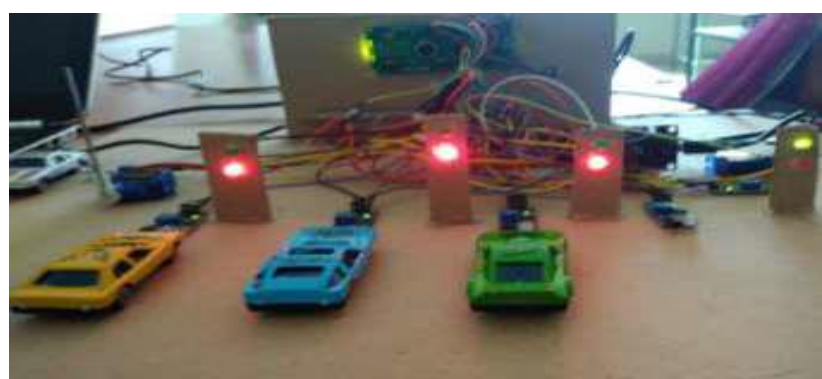

Fig.10.After reaching to the slot

\section{Stage 4:}

Before the parking zone, there will be an LCD show that is utilized to show the status of the free spaces, regardless of whether the parking slot is accessible or not.

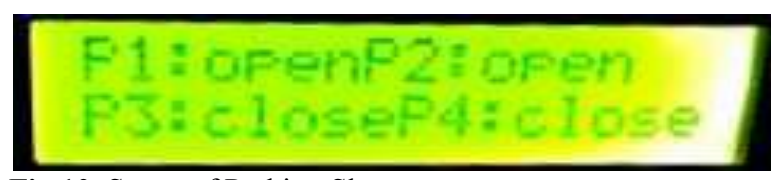

Fig.10. Status of Parking Slots

\section{APPLICATION}

The system is used to park the vehicle only in parking areas such as nearby hospitals, schools, colleges, shopping malls, etc.

\subsection{EMBEDDED SYSTEM}

Embedded approaches, as the name suggests, something that is connected to something else. For example, a fixed framework has segments with equipment, application programming and has a RealTime Operating System (RTOS) that oversees the programming of the application and provides a tool to allow the processor to run a scheduled procedure by following an arrangement to control latencies.[8].We used Arduino in this project, which is an open source method. The equipment reference plans are accepted and available on the Arduino site under a Creative Commons Attribution-Share-Alike 2.5 permit.[9].The official Arduino name strategy repository emphasizes that the task is available to consolidate other people's work into the official piece. RFID Block Schematic A streamlined square schematic of an RFID tag appears in the graph beneath. Different segments of the tag areas appeared. Regularly, the antenna apparatus is outside to the label chip and huge. [10]

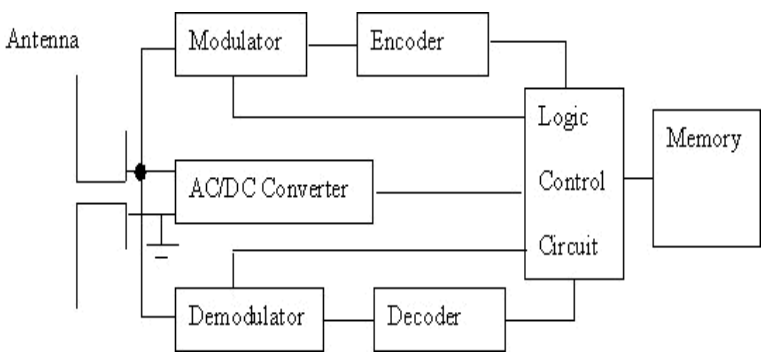

Fig.11. RFID Block Diagram

3.1.1 SERVO MOTOR GENERAL PRODUCTION 
Servomotors are not a particular type of engine, but servo motors are frequently used to refer to a motor suitable for use in a system of closed-loop control[13]. To provide position and speed feedback, the motor is paired with some type of encoder. Only the position is determined in the simplest case. Compared with the command location, the calculated location of the output is the external input to the controller. An error signal is produced if the output position varies from that expected, which then causes the motor to rotate in either direction as needed to carry the output shaft to the appropriate position.[11]. The error signal decreases to zero as the positions approach, and the motor stops.

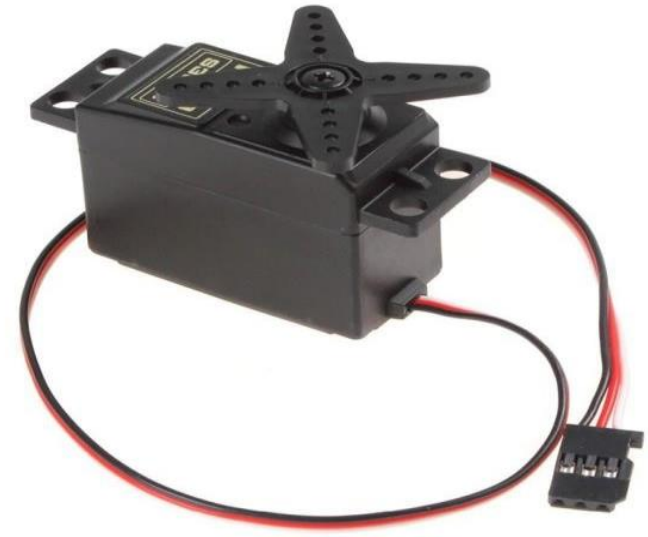

\section{CONCLUSION}

This paper proposed an IoT-based smart parking system that can provide more than just information about vacant space but also help the driver to locate an available parking slot to reduce the issue of traffic in the parking area. This smart parking system is designed using IoTbased hardware, software, and mobile application; the driver can easily check parking information and use mobile payment to pay the parking fee[13] The goal of our study is to improve the parking process by reducing the time that is required to park a car. To the best of our knowledge for providing correctly function services to the user.

\section{References}

1.Y. Huang and G. Li, "Descriptive Models for Internet of Things," in IEEE International Conference on Intelligent Control and Information Processing (ICICIP), August 2010.

2.J. Zheng, D. Simplot-Ryl, C. Bisdikian, and H. Mouftah, "The Internet of Things," in IEEE Communications Magazine, Volume:49, Issue: 11, pp:30-31, 2011.

3.O. Dokur, S. Katkoori, and N. Elmehraz, "Embedded system design of a real-time parking guidance system", Annual IEEE Systems Conference (SysCon), Orlando, FL, 2016, pp. $1-8$.

4.Anderson Ar. RubemKa, Gustavo Gi, ItamirFilho, Kayo Goncalves., "Reliability Analysis of an IoT-Based Smart Parking Application for Smart Cities", IEEE International Conference on Big Data (BIGDATA), 2017.

5.Ledalla, S. \& Mahalakshmi, T.S.. (2019). Sentiment analysis using legion kernel convolutional neural network with LSTM. International Journal of Innovative Technology and Exploring Engineering. 8. 226-229.
6.Azeem Uddin, "Traffic congestion in Indian cities: Challenges of a rising power", 23 March 2009.

7.Ledalla, Sukanya \& Mahalakshmi, Tummala. (2018). Multilingual Sentiment Analysis of Hinglish Tweets. Indian Journal of Public Health Research \& Development. 9. 1627. 10.5958/0976-5506.2018.02092.2.

8.Bagula, Antoine, Lorenzo Castelli, and Marco Zennaro. The Design of Smart Parking Networks in the Smart Cities: An Optimal Sensor Placement Model. Open Access Sensors 15 (2015): 15443-15467.

9.Kianpisheh, N. Mustaffa, P. Limtrairut, and P. Keikhosrokiani, "Smart Parking System (SPS) Architecture Using Ultrasonic Detector", 2012.

10. Y. Liu, D. Wei, N. Zhang, M. Zhao "Vehicle-license-plate recognition base on neural network," IEEE International Conference on Information and Automation, pp. 363 - 336, 2011.

11. Gibbons, P.B., Karp, B., Nath, S. andSeshan S. IrisNet: an architecture for a worldwide sensor Web. IEEE Pervasive Computing, 2(4):22 - 33, 2003.

12. Lynch J. P. andLoh. K. A Summary Review of Wireless Sensors and Sensor Networks for Structural Health Monitoring. Shock and Vibration Digest, Sage Publications,38(2):91-128, 2005.

13. Ledalla, Sukanya \& Mahalakshmi, Tummala. (2018). Multilingual Sentiment Analysis of Hinglish Tweets. Indian Journal of Public Health Research \& Development. 9. 1627. 10.5958/0976-5506.2018.02092.2.

14. K. Prasanna Lakshmi, et al. "A Study on Internet of Things with Block chain Technology ",in IEEE 3rd International Conference on Trends in Electronics and Informatics, pp:502-506, March 2019, ISBN: 978-15386-9438-1, Tirunelveli, India.

15. K. Prasanna Lakshmi, et al. "A survey on different trends in data streams" in IEEE International Conference on Networking and Information Technology (ICNIT 2010), 11-12 June 2010, pg: 451455, Manila, Philippines with ISBN: 978-1-42447577-3 sponsored by International Association of Computer Science and Information Technology (IACSIT). 